\title{
Revisión bibliográfica sobre la circulación de ideas urbanas en América Latina y el Caribe
}

Ángela-María Díaz-Márquez. Universidad de Las Américas, Quito, Ecuador.

RESUMEN | Las ciudades de América Latina y el Caribe comparten una historia común de influencia de ideas urbanas externas. Estas ideas, consideradas vanguardistas o pioneras, en la mayor parte de los casos fueron concebidas desde la experiencia europea y norteamericana, y aplicadas incluso de manera simultánea en varias ciudades a lo largo de la región. Adrián Gorelik, Arturo Almandoz, Alicia Novick y Guillermo Jajamovich son los autores cuyos trabajos han sido tomados para comenzar a construir una referencia bibliográfica con capacidad de orientar el estudio de la circulación de ideas urbanas en la región.

PALABRAS ClAVE | historia urbana, sociedad del conocimiento, urbanismo

ABSTRACT | Latin American and Caribbean cities share a common history regarding the influence of foreign urban ideas. These ideas, categorized as pioneering or avant-garde, in most cases were conceived in relation to European and North American experiences, and implemented even simultaneously in several cities across the region. Adrian Gorelik, Arturo Almandoz, Alicia Novick and Guillermo Jajamovich, are the authors whose works have been selected to build a bibliographic reference able to guide the flow of urban ideas studies in the region.

KEYWORDs | urban history, knowledge society, urbanism 


\section{El reto de la circulación de ideas urbanas en Latinoamérica}

En las principales ciudades de América Latina y el Caribe (ALyc), las tramas, el paisaje urbano, el uso de suelo, las figuras o instrumentos de planeamiento, las estructuras de gestión, los sistemas normativos e incluso la enseńanza, han sido definidos en medio de una continua dinámica de influencia urbana externa. Esta realidad ha generado gran diversidad de análisis y críticas, que abarcan desde sus potencialidades, hasta su cuestionamiento según determinadas líneas de pensamiento (modernista, identitaria, progresista o culturalistas). Eduardo Devés Valdés (1997) analizó cómo el pensamiento modernizador (exógeno) ha sido acentuado en algunos momentos, como 1850, 1890, 1940, 1985, mientras el identitario (endógeno) lo ha sido en otros, como 1865, 1910, 1965, en una dinámica casi cíclica. Pero (1) ¿para qué estudiar esta dinámica? y (2) ¿por dónde empezar?

(1) Actualmente, el incremento de los circuitos de transferencia de ideas urbanas bajo una lógica de diseminación podría derivar en la implementación acrítica de herramientas consideradas vanguardistas, bajo una falsa premisa de modernización. (2) Para comprender esta dinámica y su evolución, el estudio de las herramientas, adaptación y resultados debe contemplar el contexto de aplicación -local, nacional e incluso internacional-, involucrando otras líneas de pensamiento que coadyuvaron en dicho proceso. Construir una bibliografía de referencia para dibujar esta dinámica con nitidez, es un primer paso para responder ambas preguntas con mayor profundidad, entendiendo que su abordaje demanda una visión sistémica.

\section{Construcción de la revisión bibliográfica}

Para construir esta referencia bibliográfica se realizó un primer acercamiento a través de autores clave que mostraron los procesos que daban cuenta de la dinámica de estudio. Los principales fueron Adrián Gorelik y Arturo Almandoz, cuyos trabajos han mostrado diversos momentos de la influencia urbana externa, revelando la complejidad disciplinar de la dinámica. Por su parte, Alicia Novick (2009) y Guillermo Jajamovich (2013) han realizado dos estudios excelentemente documentados que ampliaron la investigación, con ilustrativos ejemplos de su evolución, así como su aplicación en una experiencia concreta en Argentina. A partir de sus revisiones historicistas y análisis más críticos, se revisaron varios de los trabajos citados por estos autores. Se incluyeron, entonces, otras disciplinas que dieron cuenta de esta dinámica, cuyas aportaciones fueron consideradas en la construcción de una visión más amplia del tema en cuestión. La información bibliográfica resultante ha sido resumida en siete periodos: (1) Inicios siglo $\mathrm{xx}$, como un capítulo introductorio; (2) 1920-1950; (3) 1950-1970; (4) 1970-1980; (5) 1980-1990; (6) 1990-2000; y (7) 2000-2010.

Adrián Gorelik: Analizó cómo fue pensada la ciudad y cuáles características de ese pensamiento impactaron las transformaciones urbanas latinoamericanas. Observó la ciudad latinoamericana como "el producto más genuino de la modernidad occidental, (...) un producto creado como una máquina para inventar la modernidad, 
extenderla y reproducirla: es un artefacto ideológico de la modernidad" (2002a, s/p). También analizó los discursos dependentistas, planteando un debate historiográfico desde la perspectiva transnacional, para comprender la raíz de la búsqueda de la modernidad desde el espejo, reflejo o irradiación de otras realidades.

Arturo Almandoz: Estudió los paradigmas de la historiografía urbana latinoamericana desde las escuelas de pensamiento que se consolidaron. Mostró cómo se ha incorporado en la agenda hispanoamericana la transferencia de ideas urbanísticas desde los trabajos de Romero, Rama, Hardoy, Castells, Lewis Mumford, Bradford, Morse, Randle, Gutiérrez Solano, Cardoso y Faletto, Rofman, entre otros, demostrando las potencialidades de un tema incrustado en la historiografía urbana latinoamericana.

Alicia Novick: Mostró cómo a finales del siglo xx se diversificaron los términos utilizados para definir la circulación de ideas urbanas en ALyc. Términos como "transferencia”, "préstamos”, “exportación-importación”, "traducción”, "retrotransferencia”, "circulación” o "diseminación”, evidenciaron la diversidad de formatos y métodos de la dinámica. Novick también analizó la complejidad de los procesos de recepción, donde a menudo se altera la idea base como evidencia de apropiación, aunque con consecuencias imprevistas.

Guillermo Jajamovich: Puso en relieve otra interesante arista de esta dinámica: la identificación de actores involucrados y sus fricciones, donde confluyen ideas no solo desde organismos internacionales, también desde contextos nacionales de países del Primer Mundo, en su interés por posicionarse en el campo disciplinar a nivel internacional. En ese marco de disputas y/o conflicto de intereses, el autor muestra algunos factores que posibilitan la expansión de las diversas redes de técnicos y profesionales.

\section{Autores más relevantes para la dinámica de estudio según periodo analizado}

(1) Inicios siglo xx: "Disciplinar la sociedad a través de la ciudad"

Paradigmas: independencias - del modelo colonial al modelo republicano-, reestructuración económica, modelo higienista haussmanniano, del urbanismo francés al Urban Planning inglés.

A modo de introducción general, desde que los países de ALyc obtuvieron sus independencias - estando en la disposición de autodeterminar su futuro, incluyendo el de sus ciudades-, se tenía como referente los países más industrializados como modelos del desarrollo. De los inicios del siglo xx se recogen documentos que analizaban la dinámica de transferencia de modelos urbanos externos, como el caso de La higiene aplicada a la construcción de las ciudades (1909-1910), de Ricardo Larraín Bravo, sobre el debate sanitario que influenciaría las siguientes propuestas urbanas en las capitales latinoamericanas. El historiador, ensayista y letrado argentino José Luis Romero (1965), considerado uno de los grandes humanistas argentinos de mediados del siglo xx, en el libro Latinoamérica, las ciudades y las ideas analizó 
la época de la nueva "burguesía criolla ilustrada" que, después de los procesos de independencia de cada país, había hecho de las ciudades espacios de representación elitista, importando la estética formal europea y estadounidense. La mayoría de los proyectos urbanos de este período estaban basados en el Urbanismo Académico de la École des Beaux-Arts, École Polytechnique y luego del Instituto de Urbanismo de la Universidad de París, aunque a partir de 1930 el planning anglosajón tomaba fuerza (Almandoz, 2002, 2007; Gutiérrez, 1995; Lezama, 2006; Pavez, 1992; Sánchez Ruiz, 2002). Horacio Capel (2003) explicó de qué forma, desde finales del siglo XIX e inicios del $\mathrm{xx}$, los vínculos profesionales comenzaron a consolidar las bases de los procesos de influencia urbana, comprendidas entonces desde las "funciones urbanas" sobre la relación campo-ciudad.

(2) 1920-1950: Influencia en la planificación urbana en América Latina y el Caribe Paradigmas: teoría del Estado del Bienestar de Keynes, Urban Planning, funcionalismo, Movimiento Moderno, entrada de la Planificación Holística de Ingeniería Social, grandes planes urbanos.

Importantes figuras de los Congresos Internacionales de Arquitectura Moderna (CIAM) trabajaron en largas estancias para gobiernos nacionales, bajo la teoría del Estado del Bienestar (Ascher, 2004; Bueno \& Bueno, 1941; Fraser, 2000; Gutiérrez, 1992; Luque Valdivia, 2004; Mattos, 2004; Mogollón, 2004; Pérez Oyarzún, 1991). Para 1940, el antropólogo Fernando Ortiz Fernández (1983) acuñó el término "transculturación", utilizado para sostener la circulación internacional de ideas; defendió la imposibilidad de repetir exactamente una componente cultural dentro de otra cultura (analizando el caso de Cuba). Posteriormente, la Escuela de Chicago postuló "la hipótesis metropolitana como problema de dominación ecológica” (Bogue, 1949/1974, citado por Capel, 2003, p. 32) que, desde el símil de la ecología, indicaba que naturalmente existen organismos de dominación y dominados. El arquitecto, historiador, intelectual y académico norteamericano Richard Morse, considerado una de las figuras más importantes sobre cultura latinoamericana, en 1957 estudió el paso de las estructuras coloniales hacia la modernización de un incipiente orden industrial, como "arenas culturales", "periferias creativas" o "cultura de mezcla". Cuestionó la noción centro-periferia, apuntando que la modernización fue una dinámica abierta donde se añadieron elementos singulares (locales) que generaron nuevos resultados. Para esta época, Pierre Bourdieu (2002) señaló que "le fait que les textes circulent sans leur contexte (...) est générateur de formidables malentendus", refiriéndose a los requisitos y/o limitaciones sociales de la circulación de ideas. Analizando las comunicaciones intelectuales entre Francia y Alemania después de la Segunda Guerra Mundial, observó dos retos fundamentales: "el contexto de recepción" y "las estrategias de poder" que subyacen en el acto de traducción (Novick, 2009). Bourdieu proponía "problematizar" la cuestión mediante la creación de un campo de investigación, en vez de reducirla a la simple dependencia cultural (Jajamovich, 2013). 
(3) 1950-1970: La ciudad latinoamericana, claroscuros del desarrollo dependiente Paradigmas: Guerra Fría, teorías del desarrollo, polos de desarrollo, teoría de la dependencia, continúa el Movimiento Moderno, incremento de la Planificación Holística de Ingeniería Social.

En este escenario, Leopoldo Zea (1953) analizó las posturas que defendían lo oportuno de independizarse de "la corrompida cultura occidental" e iniciar una cultura que pueda llamarse propia, aunque desde el reflejo de Norteamérica y no desde Europa. Mientras, el economista británico (1915-1990) y premio Nobel de Economía Arthur Lewis, en 1955 introducía la definición de "modelo económico dual", enfatizando en 1961 y 1979 que la región era objeto de una "exudación cultural y monopolio de creatividad" como base de un proceso de globalización, que necesita las carencias de regiones posindustriales para ser justificado. Jorge Enrique Hardoy, arquitecto, máster en Planificación Urbana y Regional y Ph.D, Universidad de Harvard, junto a Richard P. Schaedel y Richard Morse, entre otros, desde 1966 hasta los años ochenta se interesaron en los cambios disciplinares que supondría el acelerado proceso de urbanización en ALyc -entre los cuarenta y los setenta- e iniciaron los simposios llamados El proceso de urbanización de América Latina desde sus origenes hasta nuestros días, en el marco de la celebración de los Congresos Internacionales de Americanistas (ICA, siglas en inglés). Para esta época, John Friedman (1968) afirmó que el futuro de América Latina hasta cierto punto era susceptible de elegirse deliberadamente desde otras arenas (Gorelik, 2008). Por tanto, en medio de los procesos de intercambio entre prestigiosos especialistas europeos y estadounidenses, surgió con fuerza la hipótesis de la "subordinación cultural". Mientras, Celso Furtado, doctor en Derecho brasileńo, uno de los principales economistas y pensadores sociales de Latinoamérica, propuesto en 2003 para el Nobel por la Asociación de Economistas Latinoamericanos, en 1969 introdujo por primera vez el término "dualismo estructural", analizando los sistemas económicos integrados por un sector "moderno" con una predominante representación de capital extranjero vinculado al sector exportador, y un sector "atrasado" con una predominante representación de actividades locales artesanales de baja productividad.

\section{(4) 1970-1980: Estándares operativos de la influencia urbana externa}

Paradigmas: neoliberalismo, sistemas mundiales, límites del crecimiento, planificación del desarrollo, regionalización.

Las venas abiertas de América Latina de Eduardo Galeano (1971) mostró cómo la división internacional del trabajo básicamente consistía en unos países que se "especializan en ganar y otros en perder”. Bajo esa máxima, ALyc se especializó en perder desde la colonización hasta entonces: "la región sigue trabajando de sirvienta" al servicio de necesidades ajenas, señaló. Enrique Browne y Guillermo Geisse (1971) analizaron que muchos planificadores urbano-regionales de la época eligieron un rol de "neutralidad aceptada" o "marginación voluntaria", situación en que, asumiendo una suerte de "subordinación cultural", importaban modelos vanguardistas que les permitieran una legitimación profesional externa. Marcos Kaplan (1972) explicaba la naturaleza de la dependencia desde el dualismo estructural (de Celso Furtado), las relaciones económicas internacionales, así como las estructuras sociales, culturales 
y políticas urbanas. Definió la dependencia como una relación que implica "dos órdenes de fuerzas, de formas y de dinámicas" que interactúan de forma permanente y desigual. En 1973, Manuel Castells -junto a Paul Singer, Lelio Mármora, Milton Santos, Jacqueline Weisslitz, Aníbal Quijano, Ramiro Cardona, Humberto Muñoz, Orlandina de Oliveira, Claudio Stern, Fernando H. Cardoso, Cándido P. Ferreira de Camargo, Lucio Kowarick, Martha Schteingart, Horacio Torres, Enrique Browne C. Guillermo Geisse G. Gabriel Pumarino, Rosemond Cheetham, Franz Vanderschueren, el Centro Interdisciplinario de Desarrollo Urbano y Regional (CIDU), Jorge E. Hardoy, Oscar A. Moreno y Patricio Vélez- presentó Imperialismo y urbanización en América Latina, mostrando cómo conversan las influencias externas con los tomadores de decisiones y estos últimos con el contexto local. Abordó la urbanización dependiente, la acumulación primitiva, el capitalismo competitivo y el capitalismo monopolista-imperialismo. Aníbal Pinto (1973) profundizó en el "dualismo estructural" de Furtado, estableciendo la "heterogeneidad estructural", donde la ciudad era producto de esas dualidades. Roberto Schwarz (1992) transportó al ámbito de la cultura las premisas de la teoría de la dependencia con "As idéias fora do lugar", refutando la creencia de que bastaría que alyc rompiera con los "ropajes extranjeros" para encontrar la "verdadera esencia interior" (Paltti, 2002). Mientas este dilema teórico se sucedía, Roberto Matthews y Takeo Kuroko (1973) advertían sobre la baja capacidad creativa en ALyc para lograr una mayor eficiencia en la transferencia de conocimientos tecnológicos. Incluso la Iglesia católica era permeada desde la Teología de la Liberación en esta dinámica, al señalarse que "la modernidad occidental había descubierto los medios para lograr el desarrollo económico, político y social de los pueblos, como se podía ver en el Norte” (Silva, 2009, p. 94).

\section{(5) 1980-1990: Dinámica de influencia urbana en la "década perdida"}

Paradigmas: nuevo orden económico internacional, globalización, década perdida, teorías del desarrollo humano, entrada del marketing urbano y la planificación estratégica urbana, modernización del Estado e inicio de la descentralización del Estado en ALyc.

El crítico uruguayo, catedrático influyente en la academia norteamericana, Ángel Rama, influenciado por la obra de José Luis Romero (años sesenta), abordó la dimensión continental latinoamericana y las influencias culturales foráneas utilizando la noción de "transculturación". Mostró cómo paulatinamente se gestaba un pensamiento nuevo por encima de la simple recepción de ideas exógenas, y ello a partir de una conexión histórica que fusiona ideas creando un elemento nuevo nutrido de varias fuentes. Jorge Francisco Liernur (1986) afirmó: "La solución de esta disputa -en la que se identifican parcialmente las distintas fuerzas socioculturales actuantes en sede periférica- radica, entre otras razones, en la capacidad de tales fuerzas de transformar su parcialidad cultural de origen externo en sentido común interno. En esta vinculación hay producción, creación de algo que previamente no existía" (p. 62). Roberto Schwarz (1986) también reconocía el reduccionismo del dependentismo y la unilateralidad de los planteamientos centro-periferia. Analizando el contexto cultural brasileño, exponía que "la idea de copia discutida aquí opone lo nacional a lo extranjero y lo original a lo imitado, oposiciones que son irreales y 
no permiten ver la parte de lo extranjero en lo propio, la parte de lo imitado en lo original, y también la parte original en lo imitado" (p. 22). Jorge Enrique Hardoy (1988) volvía a revisar la historiografía urbana de la región con nuevos términos, como "urbanismo finisecular", "modernidad apropiada" o "modernidad adaptada". El autor destacó algunos puntos de encuentro entre las posiciones progresistas y las culturalistas.

(6) 1990-2000: Expansión: auge de ciudades intermedias en la dinámica de influencia urbana

Paradigmas: reestructuración económica en ALyc (Consenso de Washington), Índice de Desarrollo Humano, teoría del desarrollo a escala humana, gobernabilidad; se consolidan el New Public Management, City Marketing y la Planificación Estratégica Urbana.

Para los años noventa, Néstor García Canclini, con su trabajo Culturas híbridas (1997), explicó la hibridez constitutiva de la multiculturalidad en grandes ciudades de Alyc, en un momento en que el concepto de globalización estaba en pleno auge, explicando los llamados Sistemas Mundiales. Mientras, Anthony King (1990), con Global Cities, hizo un paralelismo entre globalización-colonización desde el análisis histórico de ambos procesos y cómo en los años noventa se establecieron los criterios que posicionaban ciudades globalmente (Almandoz, 2008b). Mike Savage y Alan Warde (1993) publicaron Urban Sociology, Capitalism and Modernism, donde discutían sobre los desequilibrios de la división internacional del trabajo, las repercusiones de la desindustrialización del Primer Mundo desplazándola al Tercero, cuyas ciudades fueron el escenario de las tensiones culturales de la modernidad. Mark T. Berger (1995) publicó Under Northern Eyes. Latin American Studies and United States Hegemony in the Americas, 1898-1990, sobre el desarrollo intelectual de los Estados Unidos y su influencia en Latinoamérica, a partir de los estudios de Antonio Gramsci sobre las teorías de la modernización, la dependencia y los Sistemas Mundiales. Peter Hall (1996) escribió Ciudades del mañana. Historia del urbanismo del siglo $X X$, mostrando que uno de los principales factores de esta dinámica residía en la circulación de ideas mediante una lógica de diseminación multicanal. En 1995, en tanto, Christiane Crasemann Collins observó la retrotransferencia que se daba en este proceso, donde los expertos internacionales también recibían del contexto aprendizajes que influían en su concepción disciplinar (Novick, 2009). Luiz Cesar de Queiroz Ribeiro y Robert Moses Pechman (1996) recogieron los trabajos del seminario Origens das Politicas Urbanas Modernas: Europa, América Latina, Empréstimos e Traduçóes (1994), que describían la llegada de profesionales e instituciones extranjeras que participaban en la implementación de ideas urbanas en ciudades brasileńas; utilizaron términos como "transferencias, préstamos y traducciones" para evidenciar una dinámica capaz de generar algo nuevo (Fernandes, 1998). Para 1997, Néstor García Canclini publicó "Culturas híbridas y estrategias comunicacionales", donde se preguntaba "qué utilidad puede tener la noción de hibridación al aproximarnos al fin de siglo y en medio de la radical recomposición de los mercados y las fronteras culturales". La hibridación cobraba sentido en una constelación de conceptos como "modernidad-modernización-modernismo, diferencia-desigualdad, heterogeneidad 
multitemporal, reconversión”. Alonso de Toro (1999), en sus estudios para ubicar la "teoría de la cultura" dentro y fuera de Latinoamérica, alude al "sincretismo" como un estado, y a la "heterogeneidad" e "hibridez" como estrategia, analizando la imitación-apropiación-reapropiación sobre el vínculo cultural centro-periferia como un "logocentrismo europeo-occidental".

(7) 2000-2010: Ciudades como objeto y sujeto en la dinámica de influencia urbana Paradigmas: neoestructuralismo de la Comisión Económica para América Latina y el Caribe (CEPAL), sostenibilidad (paradoja de Jevons, capitalismo verde, etcétera), urbanismo ecológico, cambio climático, gobernabilidad, de la planificación estratégica urbana al pensamiento estratégico; surgen los Policy Transfer Studies.

Silviano Santiago (2000), con su obra El entrelugar del discurso latinoamericano, sitúa la periferia en el centro, invirtiendo el diagnóstico que le asigna el papel de copia degradada del original. Describe la interacción entre dos civilizaciones ajenas entre sí, cuyo primer encuentro se enmarca en la ignorancia mutua, donde una se impone sobre otra renombrándola, reestructurándola y redefiniéndola, pero en un proceso bidireccional. Joe Nasr y Mercedes Volait (2003) analizaron la influencia del urbanismo europeo y estadounidense en otras ciudades del mundo, donde la transferencia puede ser coercitiva o voluntaria, dependiendo de las circunstancias (Novick, 2009). Roberto Monteiro de Andrade (2004) utilizó el término "resonancia” para nombrar la circulación de las ideas a través de la diseminación. Dichas resonancias aluden a una diseminación difusa, como mostró Jean-Louis Cohen en 1995 o Angotti Salgueiro en 2004 (Novick, 2009, p. 11). Gilbert M. Joseph (2005) abordó el viaje de ida y vuelta en la cultura latinoamericana en su relación con los Estados Unidos, entendiendo que más que una transferencia unidireccional de estilos de vida y pensamiento, se generaba una nueva cultura trasnacional. Jordi Borja (2007) abordó el flujo internacional de la circulación de ideas urbanas desde las llamadas ciudades globales, donde se descontextualiza la "ciudad" relegada por la "urbanización”, mermando la importancia de los hechos políticos, económicos, sociales, culturales y físicos que le dan sentido. Emilio José Luque Azcona (2008), en su trabajo "Globalización y ciudad: la reinvención de espacios urbanos en América Latina”, analizó algunos efectos de la globalización -como fenómenoen las ciudades de Alyc, observando los grandes proyectos dirigidos por gobiernos locales para atraer inversiones mediante el city marketing norteamericano y europeo,

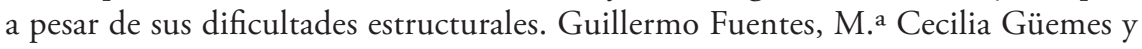
Carolina Isaza (2009) también analizaron la copia acrítica de herramientas vanguardistas externas, que, amparándose en la democratización y modernización del Estado, hicieron las administraciones públicas locales de ALyc.

\section{¿En qué punto estamos y hacia dónde se dirige el discurso? (2010-2015)}

En 2014, Alonso de Toro, con su trabajo "Hacia una teoría de la cultura de la hibridez como sistema científico transrelacional, transversal y transmedial”, profundiza desde el plano cultural en la "transculturalidad", indicando que no implica la pérdida de lo propio o la homogenización de la cultura. Víctor Delgadillo, también 
en 2014 con su trabajo "Urbanismo a la carta: teorías, políticas, programas y otras recetas urbanas para ciudades latinoamericanas", observa un proceso de "exudación cultural y monopolio de creatividad” (como explicó Lewis en 1961 y 1979), señalando cómo una serie de ideas urbanas externas circularon de manera acrítica como un menú -"recetas probadas"- por las ciudades latinoamericanas.

Figura I | Línea de tiempo sobre algunos de los autores, teorías y contextos (modelos, herramientas y paradigmas) más relevantes de cada etapa estudiada

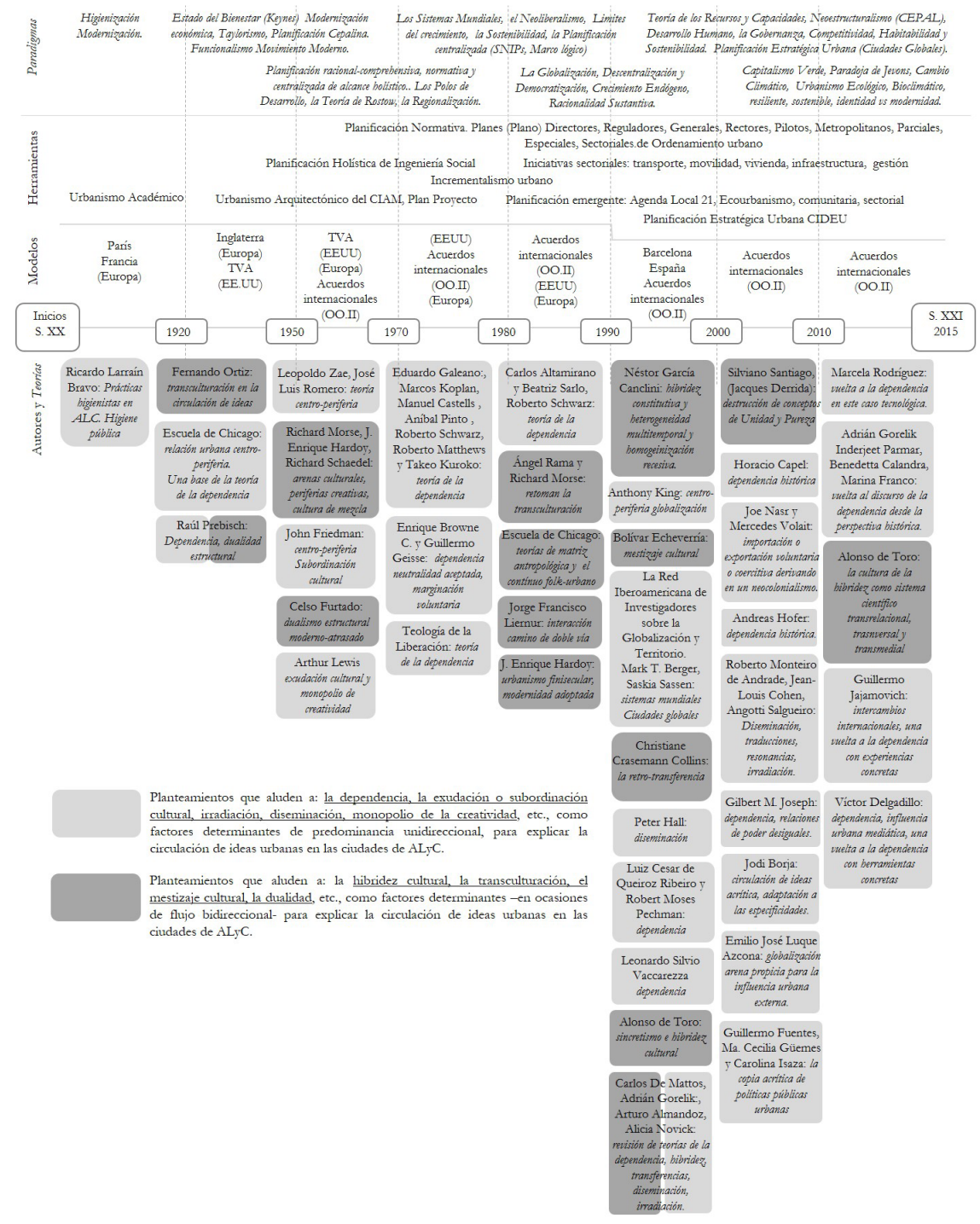


A pesar de que la hibridez cultural es evidente, persiste un vacío en reconocer que las culturas implicadas tienen pesos dispares en el campo disciplinar en cuestión. Una muestra de esto está en los resultados de los primeros planes estratégicos transferidos desde la experiencia de Barcelona. Estos planes no fueron permeados suficientemente por los condicionantes locales correspondientes; más bien, reflejaron que dentro del indudable mestizaje cultural, prevaleció una valoración mayor de los nuevos elementos de procedencia externa. Entre los trabajos de Toro y de Delgadillo -así como en otros de las etapas anteriores- hay una evidente inconsistencia, que no necesariamente es el fruto de una contradicción; es posible que la falta de estudios vinculados a ambas disciplinas genere tal ambigüedad.

\section{Conclusión}

Tal y como se observa en la figura 1, algunos autores han estudiado las ciudades de América Latina y el Caribe desde la influencia de modelos urbanos externos; otros lo han hecho registrando la heterogeneidad e interculturalidad de las ciudades de la región, evidenciada en este proceso de interacción con Europa y Estados Unidos de manera histórica. Sus puntos de vista no siempre han sido una constante discursiva, aunque sí se puede apreciar cómo dos discursos diferenciados se entremezclan frecuentemente: (1) la búsqueda permanente de la modernidad en el espejo, reflejo o irradiación de otros contextos: "la dependencia"; y (2) la búsqueda de la identidad como explicación a ese constante lazo con otras latitudes: "la hibridez cultural".

Para orientar el estudio de la circulación de ideas urbanas en ALyc, es recomendable considerar de partida tres retos, dos de ellos probablemente con potencial para estudiarse de forma objetiva a través de técnicas o métodos parametrizados; y uno empírico que se podría interpretar como subjetivo, pero que se reitera en varios estudios consultados. En una experiencia concreta de circulación de ideas urbanas es necesario:

1. Establecer las variables que responden a una dependencia disciplinar: por ejemplo, en la estructura, tecnología, gestión y recursos (económicos, humanos, administrativos, legales, etcétera) que demandan de los instrumentos que se importan, versus la realidad local y sus propios procesos.

2. Establecer las variables que responden a la hibridez cultural de la región: por ejemplo, en las tendencias de cambio en los usos, significados, apropiaciones, redefiniciones, etcétera, de la ciudad y sus diversos espacios.

3. Por último (reto subjetivo, expuesto a la interpretación de cada lector en su contexto), partir desde un posicionamiento crítico, que supere la "neutralidad aceptada" o "marginación voluntaria", bajo una "subordinación cultural" (Browne $\&$ Geisse, 1971). Este último punto es crucial, puesto que no es deseable ser (o sentirse) objeto de un proceso de subordinación cultural; pero, según la insistencia de los documentos revisados, pareciera que es fácil caer en la justificación de una variable que técnicamente obedece a un estado de dependencia a través de la hibridez cultural, para ocultar que efectivamente hay una subordinación cultural. 
Es necesario continuar documentando, analizando y parametrizando (en la medida de lo posible) la dinámica de circulación de ideas urbanas en la región. Un desafío complejo y no resuelto, que revela la vigencia del dilema planteado por Browne y Geisse más de cuarenta años después.

\section{Agradecimientos}

A José Miguel Fernández Güell, Duyot (ETSAM - UpM), AuiA. A las familias Muelas Romero y Muelas Díaz. A Adrián Gorelik, Arturo Almandoz, Alicia Novick y Guillermo Jajamovich. Y a la Dirección General de Investigación de la Universidad de Las Américas en la persona de su directora, la Dra. Tannya L. Lozada.

\section{Referencias bibliográficas}

Almandoz, A. (2002). Notas sobre historia cultural urbana. Una perspectiva latinoamericana. Perspectivas Urbanas / Urban Perspectives, 1, 29-39. En https:/upcommons.upc.edu/ handle/2099/20

Almandoz, A. (2003). Historiografía urbana en Latinoamérica: Del positivismo al postmodernismo. Diálogos, 7(1), 117-156. En http://www.dialogos.uem.br/index.ph p?journal=ojs\&page=article\&op=view\&path $\% 5 B \% 5 \mathrm{D}=214$

Almandoz, A. (2007). Modernización urbanística en América Latina. Luminarias extranjeras y cambios disciplinares 1900-1960. Iberoamericana, 7(27), 59-78. En http://www.iai. spk-berlin.de/fileadmin/dokumentenbibliothek/Iberoamericana/2007/27-Almandoz. pdf

Almandoz, A. (2008a). Despegues sin madures. Urbanización, industrialización y desarrollo en la Latinoamérica del siglo xx. EURE, 34(102), 61-76. https://doi.org/10.4067/ S0250-71612008000200004

Almandoz, A. (2008b). Para una reseńa bibliográfica sobre la globalización urbana. Bifurcaciones, (7). En http://www.bifurcaciones.cl/2008/04/para-una-resena-bibliografica-de-laglobalizacion-urbana/

Ascher, F. (2004). Los nuevos principios del urbanismo: el fin de las ciudades no está a la orden del día. Madrid: Alianza.

Berger, M. (1995). Under Northern Eyes. Latin American studies and United States hegemony in the Americas, 1898-1990. Bloomington, In: Indiana University Press.

Bogue, D. J. (1949/1974). La estructura de la comunidad metropolitana. En G. A. Theodorson (comp.). (1974), Estudios de ecología humana (vol. II, pp. 337-360). Barcelona: Labor.

Borja, J. (2007). Revolución y contrarrevolución en la ciudad global: las expectativas frustradas por la globalización de nuestras ciudades. EURE, 33(100), 35-50. https://doi. org/10.4067/S0250-71612007000300003

Bourdieu, P. (2002). Les conditions sociales de la circulation internationale des idées. Actes de la recherche en sciences sociales, 145(1), 3-8. https://doi.org/10.3406/arss.2002.2793

Browne, E. \& Geisse, G. (1971). ¿Planificación para los planificadores o para el cambio social? EURE, 1(3), 11-26. En http://www.eure.cl/index.php/eure/article/view/823 
Bueno, A. C., \& Bueno, J. C. (1941). Alguns aspectos dos problemas de urbanismo. Tese apresentada ao I Congresso Brasileiro de Urbanismo. Urbanismo e Viação, 4,15: 16:33-34.

Capel, H. (2003). Una mirada histórica sobre los estudios de redes de ciudades y sistemas urbanos. GeoTrópico, 1(1), 30-65. En http://www.geotropico.org/1_1_Capel.html

Castells M. (1973). Imperialismo y urbanización en América Latina. Barcelona: Gustavo Gili.

Chaparro, J. (2007). La segregación digital en América Latina y el Caribe: reflejo de las inequidades sociales y la dependencia tecnológica. Scripta Nova. Revista Electrónica de Geografia y Ciencias Sociales, 245(23). En http://www.ub.edu/geocrit/sn/sn-24523. htm

Cohen, J. L. (1995). Scenes of the world to come: European architecture and the American collage 1893-1960. Paris: Flammarion.

Crasemann Collins, C. (1995). Urban interchange in the southern cone: Le Corbusier (1929) and Werner Hegemann (1931) in Argentina. Journal of the Society of Architectural Historians, 54(2), 208-227. https://doi.org/10.2307/990968

Delgadillo, V. (2014). Urbanismo a la carta: teorías, políticas, programas y otras recetas urbanas para ciudades latinoamericanas. Cadernos Metrópole, 16(31), 89-111. https:// doi. org/10.1590/2236-9996.2014-3104

Devés, E. (1997). El pensamiento latinoamericano a comienzos del siglo xx: la reivindicación de la identidad. CUYO, Anuario de Filosofía Argentina y Americana (14), 11-75. En http://bdigital.uncu.edu.ar/objetos_digitales/1626/devescuyo14.pdf

Fernandes, R. Braga (1998). Reseña de "Cidade, povo e nação. Gênese do urbanismo moderno", de L. C. de Q. Ribeiro y R. Pechman (orgs.). Rio de Janeiro: Civilização Brasileira, 1996 (454 p). Biblio 3w. Revista Bibliográfica de Geografia y Ciencias Sociales, 86(4 de mayo de 1998). En http://www.ub.edu/geocrit/b3w-86.htm

Fraser, D. (2000). The Welfare State. Sutton Pocket Histories. Stroud, uk: Sutton Publishing.

Friedman, J. (1968). El futuro de la urbanización en América Latina: algunas observaciones sobre el papel de la periferia. Santiago, Chile: Programa de Asesoría en Desarrollo Urbano y Regional, Fundación Ford [versión mimeografiada].

Fuentes, G., Güemes, M. C. \& Isaza, C. (2009). Modernizar y democratizar la Administración Pública. Una mirada desde la realidad latinoamericana. Enfoques: Ciencia Política y Administración Pública, 7(11), 55-84. En http://www.redalyc.org/articulo. oa?id=96011647003

Furtado, C. (1967). La teoría del desarrollo económico (6a ed., 1976). México, D.F.: Siglo Veintiuno.

Furtado, C. (1969). Laeconomia latinoamericana. Formación histórica y problemas contemporáneos. México, D.F.: Siglo Veintiuno.

Galeano, E. (1971). Las venas abiertas de América Latina (8a imp., 2009). Madrid: Siglo Veintiuno.

García Canclini, N. (1990). Culturas híbridas. Buenos Aires: Paidós.

García Canclini, N. (1997). Culturas híbridas y estrategias comunicacionales. Estudios sobre las culturas contemporáneas, 3(5), 109-128. En http://bvirtual.ucol.mx/descargables/115_ culturas_hibridas.pdf 
Gorelik, A. (2002a). Cultura urbana y pensamiento social en América Latina. [Rodolfo Giunta, Historia Cultural Urbana - Patrimonio Histórico Cultural]. Presentación en el Seminario del Centre of Latin American Studies, Cambridge, 27 de mayo 2002. En http:// rodolfogiunta.com.ar/Historia\%20urbana/Cultura\%20urbana\%20(Adrian\%20 Gorelik).pdf

Gorelik, A. (2002b). Imaginarios urbanos e imaginación urbana. Para un recorrido por los lugares comunes de los estudios culturales urbanos. EURE, 28(83), 11-26. https://doi. org/10.4067/S0250-71612002008300008

Gorelik, A. (2003). Lo moderno en debate: ciudad, modernidad, modernización. [Punto de vista en Bazar Americano. Rodolfo Giunta, Historia Cultural Urbana - Patrimonio Histórico Cultural]. En http://www.rodolfogiunta.com.ar/Historia\%20urbana/Lo\%20 moderno\%20en\%20debate\%20(Adrian\%20Gorelik).pdf

Gorelik, A. (2008). La aldea en la ciudad. Ecos urbanos de un debate antropológico. Revista del Museo de Antropología, 1(1), 73-96. En https://revistas.unc.edu.ar/index.php/ antropologia/article/view/5398

Gorelik, A. (2012). Miradas cruzadas. El viaje latinoamericano de la planificación norteamericana. 15th International Planning History Society Conference. São Paulo, 15-18 de julio 2012. En http://www.bifurcaciones.cl/bifurcaciones/wp-content/ uploads/2015/02/bifurcaciones_018_Gorelik.pdf]

Gutiérrez, R. (1992). Iberoamérica. Tradiciones, utopias y novedad cristiana. Madrid: Encuentro.

Gutiérrez, R. (1995). Jorge Enrique Hardoy: su aporte a la historia urbana de América Latina. EURE, 21(62), 9-15. En http://www.eure.cl/index.php/eure/article/view/1122

Hall, P. (1996). Ciudades del mañana. Historia del urbanismo del siglo XX. Barcelona: Ediciones del Serbal.

Hardoy, E. (1988). Teorias y prácticas urbanisticas en Europa entre 1850 y 1930. Su traslado a América Latina y Buenos Aires. Buenos Aires: Grupo Editor Latinoamericano.

Hardoy E. \& Schaedel R. P. (1975). Sistemas sociopolíticos y urbanización. Una selección de ejemplos históricos y contemporáneos. Las ciudades de América Latina y sus áreas de influencia a través de la historia (pp. 79-112). Buenos Aires: Ediciones SIAP.

Jajamovich, G. (2013). Miradas sobre intercambios internacionales y circulación internacional de ideas y modelos urbanos. Revista de Investigación Social Andamios, 20(22), 91-111. En http://www.scielo.org.mx/scielo.php?script=sci_ arttext $\&$ pid $=$ S1870-00632013000200006

Joseph, G. (2005). Encuentros cercanos. Hacia una nueva historia cultural de las relaciones entre Estados Unidos y América Latina. En R. Salvatore (comp.), Culturas imperiales. Experiencia y representación en América, Asia y África (pp. 89-120). Rosario, Argentina: Beatriz Viterbo.

Kaplan, M. (1972). La ciudad latinoamericana como factor de transmisión de control socioeconómico y político externo durante el período contemporáneo. Boletín del Centro de Investigaciones Históricas y Estéticas, 14, 90-124. Caracas: Universidad Central de Venezuela.

King, A. D. (1990). Global cities: Post-imperialism and the internationalization of London. London and New York: Routledge.

Larraín Bravo, R. (1910). La higiene aplicada en las construcciones (3 vol.). Santiago, Chile: Imprenta Cervantes. 
Lezama Hernández, E. M. (2006). Lo global y lo local en Venezuela: el proceso modernizador y su impacto en Ciudad Guayana. Tesis doctoral, Universidad de Granada, Departamento de Sociología.

Liernur, J. (1986). El discreto encanto de nuestra arquitectura 1930/1960. Summa, (223), 60-79.

Liernur, J. (2008). Trazas de futuro. Episodios de la cultura arquitectónica de la modernidad en América Latina. Santa Fe, Argentina: Universidad Nacional del Litoral.

Liernur, J. \& Pschepiurca, P. (2008). La Red Austral. Obras y proyectos de Le Corbusier y sus discípulos en la Argentina (1924-1965). Bernal, Buenos Aires: Universidad Nacional de Quilmes.

Luque Azcona, E. J. (2008). Globalización y ciudad: la reinvención de espacios urbanos en América Latina. Anuario de Estudios Americanos, 2(65), 265-287. En https://idus. us.es/xmlui/handle/11441/44065

Luque Azcona, E. M. (2008). Globalización y ciudad: la reinvención de espacios urbanos en América Latina. Anuario de Estudios Americanos, 65(2), 265-287. https://doi. org/10.3989/aeamer.2008.v65.i2.122.

Luque Valdivia, J. (2004). Consolidación y convergencia de dos tradiciones urbanas. Comunicación presentada a la 11th International Planning History Association Conference 2004. Barcelona, 14-17 de julio, 2004. Reproducido en Cuestiones Urbanas, 1 [Departamento de Urbanística y Ordenación del Territorio, Universidad de Sevilla] (2010), 15-25. En https://departamento.us.es/duot/master/archivos/2010-11/ CuestionesUrbanas1.pdf

Matthews, R. \& Kuroko, T. (1973). La transferencia de tecnología industrial extranjera a los países latinoamericanos: Características generales de problemas y sugerencias para la acción. Nueva Sociedad, (8-9 sept.-dic.), 88-98. En http://nuso.org/media/articles/ downloads/90_1.pdf

Mattos, C. de (2004). De la planificación a la governance: implicancias para la gestión territorial y urbana. Revista Paranaense de Desenvolvimiento, (107), 9-23. En http://www.ipardes. gov.br/pdf/revista_PR/107/carlos_mattos.pdf

Mogollón, L. E. (2004). Influence of foreign theories and urban models in the formulation of masters plans of Latin American cities, during the 1930-1960 period. The 11th International Planning History Conference: Planning Models and the Culture of Cities. Universitat Politècnica de Catalunya, Escuela Técnica Superior de Arquitectura del Vallès (ETSAV), Barcelona. En http://www.etsav.upc.es/personals/iphs2004/pdf/139_p. pdf

Monteiro de Andrade, R. (1994). Camillo Sitte, Camille Martin e Saturnino de Britto: traduçoes e transferencias dee idéas urbanísticas. Seminario Origens das Politicas Urbanas Modernas: Empréstimos e Traduçōes. IPPUR-UfRJ, ANPUR, CNPq, CSU-CNRS, Itamontes, Brasil.

Morse, R. (1957). La ciudad artificial. Estudios Americanos, 13(67-68) 284-293.

Morse, R. (1973). Prolegómenos a la historia urbana latinoamericana. En Richard Morse, Las ciudades latinoamericanas (pp. 9-77). México, D.F.: SepSetentas.

Morse, R. (1976). Ciudades latinoamericanas: aspectos de su función y estructura En G. Germani (comp.), Urbanización, desarrollo y modernización (pp. 241-263). Buenos Aires: Paidós. Publicado originalemente en 1960. 
Morse, R. (1978). Los intelectuales latinoamericanos y la ciudad (1860-1940). En J. E. Hadoy, R, M. Morse \& R. P. Schaedel (comps.), Ensayos histórico-sociales sobre la urbanización en América Latina (pp. 91-112). Buenos Aires: Ediciones SIAP.

Morse, R. (1985). Ciudades 'periféricas' como arenas culturales (Rusia, Austria, América Latina). En R. Morse, \& J. E. Hardoy (comps.), Cultura urbana latinoamericana (pp. 39-62). Buenos Aires: Consejo Latinoamericano de Ciencias Sociales (clacso).

Morse, R. \& Hardoy, J. E. (1985). La ciudad letrada. Cultura urbana latinoamericana (pp. 1137). Buenos Aires: Consejo Latinoamericano de Ciencias Sociales (clacso). [Versión digitalizada en http://bvsde.org.ni/clacso/publicaciones/morse.pdf]

Nasr, J. \& Volait, M. (eds.). (2003). Urbanism: Imported or exported? Native aspirations and foreign Plans. West Sussex, uk: Wiley-Academy.

Novick, A. (2009). La ciudad, el urbanismo y los intercambios internacionales. Notas para la discusión. Revista Iberoamericana de Urbanismo. Iberoamérica, espacio de oportunidades, (1), 4-13. En http://upcommons.upc.edu/handle/2099/12252

Ortíz Fernández, F. (1983). Contrapunteo cubano del tabaco y el azúcar. Serie Pensamiento Cubano. La Habana: Editorial de Ciencias Sociales.

Outtes, J. (2002). Disciplinando la sociedad a través de la ciudad. El origen del urbanismo en Argentina y Brasil (1894-1945). EURE, 28(83), 7-29. https://dx.doi.org/10.4067/ S0250-71612002008300002. Reproducido en: Disciplining society through the city: The genesis of city planning in Brazil and Argentina (1894-1945). Bulletin of Latin American Research, 22(2) (April 2003), 137-164. https://doi.org/10.1111/14709856.00069

Paltti, E. J. (2002). El problema de "las ideas fuera de lugar" revisitado. Más allá de la "historia de las ideas". Presentado en Seminario de Historia Intelectual, El Colegio de México, enero 2002. En http://shial.colmex.mx/textos/EliasPalti-Enero2002.pdf

Pavez, M. I. (1992). La institución del urbanismo en la Facultad de Arquitectura y Urbanismo de la Universidad de Chile (1928-1988). Santiago, Chile: Universidad de Chile, Facultad de Arquitectura y Urbanismo, Departamento de Urbanismo. En http://bit.ly/2u6xNzY

Pérez Oyarzún, F. (ed.). (1991). Le Corbusier y Sudamérica: viajes y proyectos. Vol. 2 de Serie Arte/ Arquitectura. Santiago, Chile: Ediciones Arq (Escuela de Arquitectura, P. Universidad Católica de Chile).

Pinto, A. (1973). Inflación: Raíces estructurales. México D.F.: Fondo de Cultura Económica.

Quijano, A. (1977). Dependencia, urbanización y cambio social en Latinoamérica. Lima: Mosca Azul.

Rama, A. (1982). Transculturación narrativa en América Latina. México, D.F.: Siglo Veinitiuno. Rama, A. (1984). La ciudad letrada. Montevideo: Fundación Internacional Ángel Rama.

Ribeiro, L. C. de Q. \& Pechman, R. (orgs.). (1996). Cidade, povo e nação. Gênese do urbanismo moderno. [Textos presentados en el seminario Origens das Políticas Urbanas Modernas: Europa, América Latina, Empréstimos e Traduçôes, realizado en Itamonte, Minas Gerais, 29 de agosto-2 de septiembre 1994]. Rio de Janeiro: Civilização Brasileira.

Rodríguez, M. (2011). Dependencia y nuevas tecnologías. Huellas, 15(18), 279-287. En http:// www.biblioteca.unlpam.edu.ar/pubpdf/huellas/v15a18rodriguez.pdf

Romero, J. L. (1965). Latinoamérica, las ciudades y las ideas. Buenos Aires: Siglo Veintiuno.

Romero, J. L. (2009). La ciudad occidental. Las culturas urbanas en Europa y América. Buenos Aires: Siglo Veintiuno. 
Sánchez Ruiz, G. (2002). El primer posgrado en planificación y urbanismo en México. Un desencuentro en la historia. Innovación Educativa, 2(7), 36-45.

Santiago, S. (2000). El entrelugar del discurso latinoamericano. En A. Amante \& F. Garramuñon (comps.), Absurdo Brasil. Polémicas en la cultura brasileña (pp.57-76). Buenos Aires: Biblos. En http://bit.ly/2uadp0V

Savage. M. \& Warde. A. (1993). Urban sociology, capitalism and modernism. Basingstoke, uK: Macmillan.

Schteingart, M. (ed.). (1973). Urbanización y dependencia en América Latina. Buenos Aires: Ediciones SIAP.

Schwarz, R. (1977). As idéias fora do lugar. En Ao vencedor as batatas (pp. 10-31). São Paulo: Livraria Duas Cidades.

Silva, S. (2009). La Teología de la Liberación. Teología y Vida, 50(1-2), 93-116. https://doi. org/10.4067/S0049-34492009000100008

Toro, A. (1999). La postcolonialidad en Latinoamérica en la era de la globalización. ¿`ambio de paradigma en el pensamiento teórico-cultural latinoamericano? En A. de Toro y F. de Toro (eds.), El debate de la postcolonialidad en Latinoamérica. Una postmodernidad periférica o cambio de paradigma en el pensamiento latinoamericano (pp. 31-77). Madrid: Iberoamericana / Frankfurt: Vervuert. En https://patriciolepe.files.wordpress. com/2013/07/la-postcolonialidad-en-latinoamc3a9rica-alfonso-de-toro.pdf

Toro, A. (2014) Hacia una teoría de la cultura de la 'hibridez' como sistema científico transrelacional, 'transversal' y 'transmedial'. Ibero-Amerikanisches-Forschungsseminar, 275-329. En http://home.uni-leipzig.de/detoro/wp-content/uploads/2014/03/ Cultura_hibridez.pdf

Vergara Ereche, M. (2003). La tecno-dependencia Informática y sociedad. Valparaíso: Universidad Técnica Federico Santa María.

Zea, L. (1953). América como conciencia. México, D.F.: Cuadernos Americanos. Versión de la Universidad Nacional Autónoma de México (UNAM), 1972, en http:// revistaliterariakatharsis.org/zea.pdf 